\title{
Near-field pressure, intensity, and wave-number distributions for active structural acoustic control of plate radiation: Theoretical analysis
}

\author{
Bor-Tsuen Wang and Chris R. Fuller \\ Department of Mechanical Engineering, Virginia Polytechnic Institute and State University, Blacksburg, \\ Virginia 24061
}

(Received 18 July 1991; revised 2 October 1991; accepted 24 April 1992)

\begin{abstract}
This paper presents a theoretical analysis of near-field time-averaged intensity and pressure distributions of actively controlled plate-radiated sound. A harmonic point force was considered as a noise source, and a piezoelectric element bonded to the plate was applied as control actuator. A single microphone that measures sound pressures in the radiated acoustic far field serves as an error sensor. The optimal input voltage to the piezoelectric actuator is obtained by minimizing a quadratic cost function, defined as the mean square of the error sensor signal. The influence of the location of the error microphone on near-field pressure and normal intensity was studied. Fourier transforms in the wave-number domain are also used to study the mechanisms of control. This work provides a better understanding of the behavior inherent in controlling structurally radiated sound using piezoelectric actuators. In particular, an understanding of the near-field behavior under closed loop control is inherent in the design of sensor arrays located near or on the plate surface.
\end{abstract}

PACS numbers: 43.40.Dx, 43.40.Rj, 43.40.Vn

\section{LIST OF SYMBOLS}

$A$

$\boldsymbol{B}$

$C_{0}$

$c$

$d_{31}$

$F$

$h$

$I_{z_{12}}$

$I_{m}, I_{n}$

$I_{m n}$

$K$

\section{$L_{x}, L_{y}$}

$N_{\text {mike }}$

$N_{s}$

$N_{c}$

$P_{m n}$

$p_{c}$

$p_{t} \quad$ total sound pressure

$p_{n} \quad$ sound pressure due to noise sources (point forces)

p optimal parameter vector

$Q_{m n} \quad$ modal function q amplitude vector of noise wave

$t$ time constant

$t_{a} \quad$ thickness of piezoceramic patch

$V \quad$ applied voltage to piezoceramic patch

$w \quad$ plate displacement

$W_{m n} \quad$ modal amplitude

$x, y, z \quad$ Cartesian coordinates of radiating field

$x_{f}, y_{f} \quad$ location of point force

$x_{1}, x_{2}, y_{1}, y_{2}$ location of piezoceramic patch

$\alpha_{m}, \beta_{n} \quad$ plate modal numbers

$k \quad \omega / c$, acoustic wave number

$k_{p} \quad$ structural primary wave number

$R, \theta, \phi \quad$ polar coordinates of radiating field

$\left(R_{i}, \theta_{i}, \phi_{i}\right)$ the $i$ th microphone coordinates

$\Delta z_{12} \quad$ distance between point 1 and 2

$\Lambda \quad d_{31} V / t_{a}$, free strain of piezoelectric element

$\lambda$ acoustic wavelength in air

$\rho \quad$ mass density of air

$\rho_{p} \quad$ mass density of plate

$\stackrel{\text { cost function }}{\Psi}$

$\omega \quad 2 \pi f$, excitation frequency

$\omega_{m n} \quad$ plate natural frequency

$\xi, \eta \quad$ plate coordinates, origin at plate corner

$\xi^{\prime}, \eta^{\prime} \quad$ transferred plate coordinates, origin at plate center

\section{Superscripts}

$f \quad$ point force noise

$c \quad$ piezoelectric actuator 


\section{INTRODUCTION}

Previous works have demonstrated the potential of multiple piezoelectric actuators as control sources in conjunction with error microphones in the far field to actively control sound radiation from structures. ${ }^{1,2}$ Wang et $\mathrm{l}^{2}$ showed the importance of properly selecting the number and location of actuators as key to efficient control of sound radiation. Their work presented far-field radiation directivity patterns as well as plate displacement distributions under open- and closed-loop control conditions, which were useful in evaluating the control performance and mechanisms. However, little work has been done on studying the nearfield pressure distributions and the radiated intensity distributions under the same conditions. These parameters are important as they provide further insight into the control mechanisms. It is also hoped to overcome the use of error microphones located in the far field by the use of sensors near or on the structure. In this context, the controlled near-field pressure should provide insight into the configurations of near-field sensors designed so as to provide reduction in farfield pressures. In this paper, "near-field" is taken to mean on or very close to the surface of the structure.

The objective of this paper is to extend the analysis presented in Ref. 2 to evaluate near-field pressure and timeaveraged intensity distributions. In order to obtain the intensity distributions, the finite difference method of Pettersen ${ }^{3}$ will be used. This method has been used successfully by a number of authors to efficiently evaluate intensity distributions in complex radiating acoustic fields. ${ }^{4,5}$ As discussed by Thompson and Tree, ${ }^{6}$ the finite difference approximation errors in acoustic intensity measurements are less than $2 \mathrm{~dB}$ from 0 to $10 \mathrm{kHz}$ for a spatial sampling separation of $8 \mathrm{~mm}$.

In this analysis, a simply supported finite plate positioned in an infinite rigid baffle was considered as the structure. The plate was excited by a steady-state harmonic disturbance in the form of an oscillating point force. The control input was applied by a piezoelectric actuator bonded to a plate surface, while error information was taken from a single microphone located in the radiated acoustic far field. Under the same control condition, the near-field pressure and time-averaged intensity distributions were evaluated, while the plate uncontrolled and controlled motion was studied in the wave-number domain.

\section{ANALYSIS}

\section{A. Plate vibration for noise and control inputs}

Figure 1 shows the arrangement and coordinates of the system. Point forces considered as noise sources were used to excite the plate, while piezoelectric actuators considered as control sources were applied to control the plate sound radiation. Under the assumption of harmonic excitation, the displacement of the simply supported plate can be written as

$w(\xi, \eta, t)=e^{i \omega t} \sum_{m=1}^{\infty} \sum_{n=1}^{\infty} W_{m n} \sin \left(\alpha_{m} \xi\right) \sin \left(\beta_{n} \eta\right)$,

where

$$
\alpha_{m}=m \pi / L_{x}, \quad m=1,2, \ldots
$$

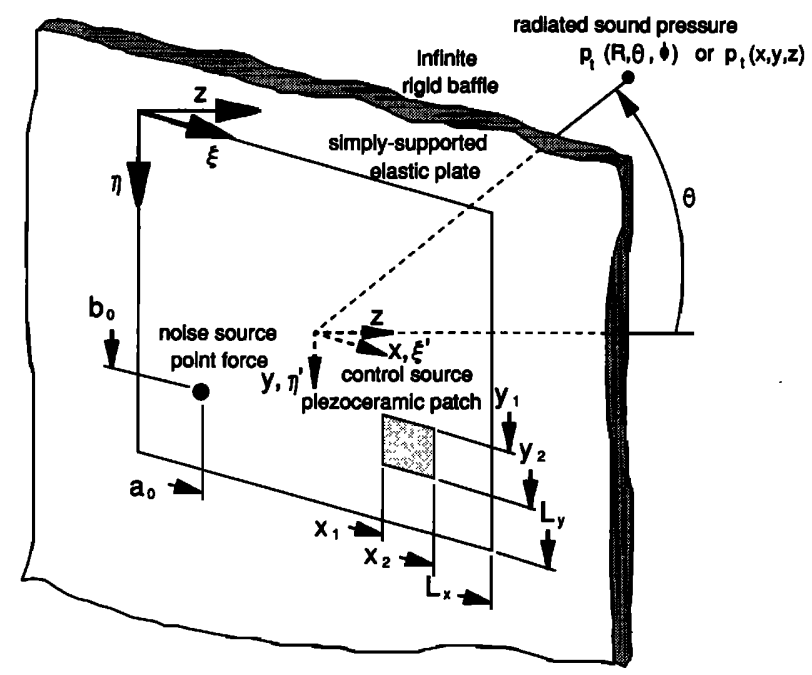

FIG. 1. Arrangement of coordinates of system.

$$
\beta_{n}=n \pi / L_{y}, \quad n=1,2, \ldots,
$$

and the amplitude $W_{m n}$ is given by

$$
W_{m n}=\frac{P_{m n}}{\rho_{p} h\left(\omega_{m n}^{2}-\omega^{2}\right)}, \quad m, n=1,2, \ldots
$$

Here, $P_{m n}$ is the modal force that depends on the exact description of the applied external load.

For the point force noise input, the modal force $P_{m n}^{f}$ is given as follows:

$$
P_{m n}^{f}=\left(4 F / L_{x} L_{y}\right) \sin \left(\alpha_{m} x_{f}\right) \sin \left(\beta_{n} y_{f}\right),
$$

where $x_{f}$ and $y_{f}$ are the coordinates of the point force actuator, $F$ is the magnitude of point force, and the superscript $f$ will signify the point force. The corresponding expression of modal force for piezoelectric excitation $P_{m n}^{c}$ was derived in Ref. 7 as

$$
\begin{aligned}
P_{m n}^{c}= & \left(4 C_{0} \Lambda / m n \pi^{2}\right)\left(\alpha_{m}^{2}+\beta_{n}^{2}\right) \\
& \times\left[\cos \left(\alpha_{m} x_{1}\right)-\cos \left(\alpha_{m} x_{2}\right)\right] \\
& \times\left[\cos \left(\beta_{n} y_{1}\right)-\cos \left(\beta_{n} y_{2}\right)\right],
\end{aligned}
$$

where $x_{1}, x_{2}, y_{1}$, and $y_{2}$ are the coordinates of the piezoelectric actuator, and the superscript $c$ will signify the piezoelectric actuator. The parameter $C_{0} \Lambda$ is defined in Ref. 7 for an actuator consisting of two identical piezoceramic patches bonded symmetrically on the two opposite plate surfaces and activated $180^{\circ}$ out-of-phase. Here, $C_{0}$ is a constant of the piezoelectric material properties and dimensions; $\Lambda=d_{31}$ $V / t_{a}$ is the strain induced in an unconstrained piezoelectric layer of thickness $t_{a}$ when a voltage $V$ is applied along its polarization direction, while $d_{31}$ is the piezoelectric dielectric strain constant.

\section{B. Sound radiation}

The radiated sound pressure is directly coupled to the plate vibration. We thus use a Rayleigh integral approach 
that relates the plate velocity to the radiated pressure as follows: ${ }^{8}$

$$
\begin{aligned}
p(R, \theta, \phi)= & \int_{\xi^{\prime}=-L_{x} / 2}^{L_{x^{\prime}} / 2} \int_{\eta^{\prime}=-L_{y^{\prime} / 2}}^{L_{y^{\prime} / 2}} \frac{j \rho \omega}{2 \pi r^{\prime}} \frac{\partial w\left(\xi^{\prime}, \eta^{\prime}, t\right)}{\partial t} \\
& \times e^{-j \omega r^{\prime} / c} d \xi^{\prime} d \eta^{\prime},
\end{aligned}
$$

where the shifted plate coordinates are

$$
\begin{aligned}
& \xi^{\prime}=\xi-L_{x} / 2, \\
& \eta^{\prime}=\eta-L_{y} / 2,
\end{aligned}
$$

and

$$
r^{\prime}=\sqrt{\left(x-\xi^{\prime}\right)^{2}+\left(y-\eta^{\prime}\right)^{2}+z^{2}} .
$$

By the substitution of Eq. (1) into Eq. (7), the radiated sound pressure can be expressed as follows:

$$
\begin{aligned}
p(x, y, z, t)= & \frac{-\rho \omega^{2}}{2 \pi} e^{i \omega t} \sum_{m=1}^{\infty} \sum_{n=1}^{\infty} W_{m n} \cdot \int_{\xi^{\prime}=-L_{x} / 2}^{L_{x} / 2} \int_{\eta^{\prime}=-L_{y} / 2}^{L_{y} / 2} \frac{e^{-j}\left[(\omega / c) \sqrt{\left(x-\xi^{\prime}\right)^{2}+\left(y-\eta^{\prime}\right)^{2}+z^{2}}\right]}{\sqrt{\left(x-\xi^{\prime}\right)^{2}+\left(y-\eta^{\prime}\right)^{2}+z^{2}}} \\
& \times \sin \left[\alpha_{m}\left(\xi^{\prime}+L_{x} / 2\right)\right] \sin \left[\beta_{n}\left(\xi^{\prime}+L_{y} / 2\right)\right] d \xi^{\prime} d \eta^{\prime} .
\end{aligned}
$$

The above integral must be evaluated numerically in the near field except for particular isolated cases of little general interest. However, an approximate closed-form solution for this integral can be obtained in the far field. Junger and Feit ${ }^{9}$ have used the stationary phase method while Roussos ${ }^{8}$ has used a solution of the Rayleigh's integral at large distances to derive a general expression for the far-field sound pressure radiated from a vibrating plate. By superposition, their analysis can be extended to describe the sound radiation from a plate excited by various noise and control sources. Thus, for $N_{s}$ noise sources (point forces) or $N_{e}$ piezoelectric actuators, the sound pressure radiated to a point, $p(R, \theta, \phi)$, in the far field can be derived as follows for noise sources:

$$
p_{n}(R, \theta, \phi)=K \sum_{j=1}^{N_{s}} \sum_{m=1}^{\infty} \sum_{n=-1}^{\infty} W_{m n j}^{f} I_{m} I_{n}
$$

for piezoelectric control excitation:

$$
p_{c}(R, \theta, \phi)=K \sum_{j=1}^{N_{c}} \sum_{m=1}^{\infty} \sum_{n=1}^{\infty} W_{m n j}^{c} I_{m} I_{n},
$$

where the constant $K$ and the quantities $I_{m}$ and $I_{n}$ can be found in Roussos ${ }^{8}$ as functions of $(R, \theta, \phi)$. Note, the above expressions hold for light fluid loading.

When the noise sources and the piezoelectric actuators act simultaneously, the resulting sound pressure field can be viewed as a superposition of the above given sound pressures for steady-state harmonic excitation. The total pressure can be conveniently written as

$$
p_{t}=p_{n}+p_{c}=\sum_{j=1}^{N_{s}} F_{j} B_{j}+\sum_{j=1}^{M_{c}}\left(C_{0} \Lambda\right)_{j} A_{j},
$$

where $B_{j}$ and $A_{j}$ are the sound pressure distribution functions for the $j$ th point force noise and $j$ th piezoelectric actuator, respectively, given by

$$
\begin{aligned}
& B_{j}(R, \theta, \phi)=K \sum_{m=1}^{\infty} \sum_{n=1}^{\infty} Q_{m n j}^{f} I_{m} I_{n}, \\
& A_{j}(R, \theta, \phi)=K \sum_{m=1}^{\infty} \sum_{n=1}^{\infty} Q_{m n j}^{c} I_{m} I_{n},
\end{aligned}
$$

where

$$
\begin{aligned}
& Q_{m n j}^{f}=W_{m n j}^{f} / F_{j}, \\
& Q_{m n j}^{c}=W_{m n j}^{c} /\left(C_{0} \Lambda\right)_{j} .
\end{aligned}
$$

\section{OPTIMAL CONTROL}

References 1 and 2 defined a suitable cost function for this application to be the integral of the mean-squared sound pressure over a hemisphere of radius $R$ in the far field. Such a cost function gives a global sense of noise reduction; however, in practical application, it is not possible to measure a continuous surface pressure. Instead, a finite number of microphones can be used to measure sound pressures in the radiating field. Therefore, the cost function to be selected here is the sum of the square of measured sound pressures at a number of points corresponding to microphone locations. Control of the noise field can be achieved by appropriately choosing the piezoelectric voltage parameters, $C_{0} \Lambda$, to minimize the selected cost function. This cost function can be written as

$$
\Psi=\sum_{i=1}^{N_{\text {mike }}}\left|p_{t_{i}}\left(R_{i}, \theta_{i}, \phi_{i}\right)\right|^{2} .
$$

When the expression for $p_{t}$ from Eq. (14) is substituted into Eq. (19), the cost function is obviously quadratic and possesses a unique minimum. A minimization procedure ${ }^{10}$ for the quadratic function was employed to calculate the optimal control parameters.

The total pressure of Eq. (14) can be expressed in vector form as

$$
p_{t}=\mathbf{B}^{T} \mathbf{q}+\mathbf{A}^{T} \mathbf{p},
$$

where the matrices $\mathbf{B}, \mathbf{q}, \mathbf{A}$, and $\mathbf{p}$ are defined in Ref. 2 with $\Lambda$ denoted as $\epsilon_{p e}$. Matrices $\mathbf{q}$ and $\mathbf{p}$ are vectors of noise input and control input strengths, respectively, while $\mathbf{B}$ and $\mathbf{A}$ are the appropriate pressure transfer terms. The cost function can then be written in matrix form as,

$$
\Psi=\mathbf{p}^{T}[\widetilde{A}] \mathbf{p}^{*}+2 \operatorname{Re}\left\{\mathbf{q}^{T}[\widetilde{B A}] \mathbf{p}^{*}\right\}+\mathbf{q}^{T}[\widetilde{B}] \mathbf{q}^{*},
$$


where matrices $[\widetilde{A}],[\widetilde{B A}]$, and $[\widetilde{B}]$ are again defined in Ref. 2.

If we let

$$
\mathbf{F}^{T}=-q^{-T}[\widetilde{B A}]
$$

then the optimal solution for the cost function can be found as in Ref. 10.

$$
\mathbf{p}_{\mathrm{opt}}=[\tilde{\boldsymbol{A}}]^{-1} \mathbf{F} \text {. }
$$

It is noted that $\mathbf{p}$ is the vector of control strengths defining the optimal voltage $\Lambda$ to each actuator.

\section{A. Time-averaged intensity}

The distribution of vectors associated with the magnitude and direction of intensity in acoustic fields have been proved useful in studying the flow of energy. The two-microphone techniques, ${ }^{3}$ which is based on the finite difference method, has been widely adopted to measure or calculate the acoustic intensity for its simplicity. The schematic used for the intensity calculation by the finite difference method is shown in Fig. 2 for the $z$ direction. The averaged acoustic pressure and particle velocity in the $z$ direction (for example) between points 1 and 2 can be formulated as follows:

$$
\begin{aligned}
& p_{12} \simeq\left(p_{1}+p_{2}\right) / 2, \\
& u_{z_{12}} \simeq-\left(p_{2}-p_{1}\right) / j \rho \omega \Delta z_{12},
\end{aligned}
$$

where

$$
\begin{aligned}
& p_{1}=\left|p_{1}\right| e^{j \phi_{1}}, \quad \phi_{1}=\tan ^{-1}\left(\frac{I_{m}\left\{p_{1}\right\}}{\operatorname{Re}\left\{p_{1}\right\}}\right), \\
& p_{2}=\left|p_{2}\right| e^{j \phi_{2}}, \quad \phi_{2}=\tan ^{-1}\left(\frac{I_{m}\left\{p_{2}\right\}}{\operatorname{Re}\left\{p_{2}\right\}}\right) .
\end{aligned}
$$

Hence, the time-averaged intensity in the $z$ direction between the two points can be shown to be

$$
I_{z_{12}}=\frac{\left|p_{1}\right|\left|p_{2}\right|}{2 \rho \omega \Delta z_{12}} \sin \left(\phi_{1}-\phi_{2}\right) .
$$

The time-averaged intensity distribution for other directions can also be derived by using a similar procedure. The total

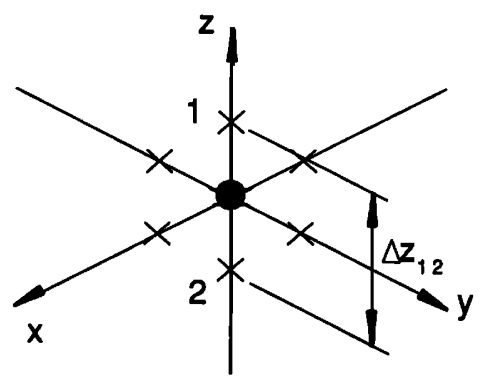

$$
\begin{gathered}
\mathrm{I}_{\mathbf{z}_{12}=} \frac{\left|\mathbf{p}_{1}\right|\left|\mathbf{p}_{2}\right|}{2 \omega \rho \Delta \mathbf{z}_{12}} \sin \left(\phi_{1}-\phi_{2}\right) \\
\mathbf{p}_{1}=\left|\mathbf{p}_{1}\right| e^{j \phi_{1}} \\
\mathbf{p}_{2}=\left|\mathbf{p}_{2}\right| e^{j \phi_{2}}
\end{gathered}
$$

FIG. 2. Schematic of intensity calculation for $z$ direction.

intensity is then the vector sum of the intensities in the $x, y$, and $z$ directions.

\section{B. Plate wave-number analysis}

The plate velocity distribution derived from Eq. (1) can be transferred to the central origin plate coordinates $\left(\xi, n^{\prime}\right)$ as

$$
\begin{aligned}
\dot{w}\left(\xi^{\prime}, \eta^{\prime}, t\right)= & j \omega e^{j \omega t} \sum_{m=1}^{\infty} \sum_{n=1}^{\infty} W_{m n} \sin \left\{\alpha_{m}\left(\xi^{\prime}+L_{x} / 2\right)\right\} \\
& \times \sin \left\{\beta_{n}\left(\eta^{\prime}+L_{y} / 2\right)\right\} .
\end{aligned}
$$

The wave-number transform of the plate velocity is then given by ${ }^{11}$

$$
\begin{aligned}
\widetilde{V}\left(\kappa_{x}, \kappa_{y}\right) & =j \omega e^{j \omega t} \int_{0}^{L_{y}} \int_{0}^{L_{x}} \dot{w}\left(\xi^{\prime}, \eta^{\prime}\right) e^{j\left(\kappa_{x} \xi^{\prime}+\kappa_{y} \eta^{\prime}\right)} d \xi^{\prime} \eta^{\prime} \\
& =j \omega e^{j \omega t} \sum_{m=1}^{\infty} \sum_{n=1}^{\infty} W_{m n} V_{m n},
\end{aligned}
$$

where

$$
\begin{aligned}
V_{m n}\left(\kappa_{x}, \kappa_{y}\right)= & \frac{4 \alpha_{m} \beta_{n}}{\left(\alpha_{m}^{2}-\kappa_{x}^{2}\right)\left(\beta_{n}^{2}-\kappa_{y}^{2}\right)}\left\{\left[\sin ^{2}\left(\frac{m \pi}{2}\right) \sin ^{2}\left(\frac{n \pi}{2}\right) \cos \left(\frac{\kappa_{x} L_{x}}{2}\right) \cos \left(\frac{\kappa_{y} L_{y}}{2}\right)\right.\right. \\
& \left.-\cos ^{2}\left(\frac{m \pi}{2}\right) \cos ^{2}\left(\frac{n \pi}{2}\right) \sin \left(\frac{\kappa_{x} L_{x}}{2}\right) \sin \left(\frac{\kappa_{y}}{L_{y}}\right)\right]-j\left[\sin ^{2}\left(\frac{m \pi}{2}\right) \cos ^{2}\left(\frac{n \pi}{2}\right) \cos \left(\frac{\kappa_{x} L_{x}}{2}\right) \sin \left(\frac{\kappa_{y} L_{y}}{2}\right)\right. \\
& \left.\left.+\cos ^{2}\left(\frac{m \pi}{2}\right) \sin ^{2}\left(\frac{n \pi}{2}\right) \sin \left(\frac{\kappa_{x} L_{x}}{2}\right) \cos \left(\frac{\kappa_{y} L_{y}}{2}\right)\right]\right\} .
\end{aligned}
$$

Hence, the plate velocity distribution can be obtained from the inverse Fourier transform

$$
\begin{aligned}
\dot{w}\left(\xi^{\prime}, \eta^{\prime}\right)= & \frac{1}{2 \pi} \int_{-\infty}^{\infty} \int_{-\infty}^{\infty} \widetilde{V}\left(\kappa_{x}, \kappa_{y}\right) \\
& \times e^{-j\left(\kappa_{x} \xi^{\prime}+\kappa_{y} \eta^{\prime}\right)} d \kappa_{x} d \kappa_{y} .
\end{aligned}
$$

The radiated power has been shown in Ref. 11 to be related to the integration of the modulus square of $\widetilde{V}\left(\kappa_{x}, \kappa_{y}\right)$. Therefore, it is of interest to evaluate the wave-number modulus spectrum of plate velocity, $\left|\widetilde{V}\left(\kappa_{x}, \kappa_{y}\right)\right|^{2}$. It is noted that the wave-number modulus spectra of modal velocity is a function of $\kappa_{x}, \kappa_{y}$ and is composed of a double infinite sum of 
modes. Only wave-number components satisfying the condition $\left(\kappa_{x}^{2}+\kappa_{y}^{2}\right)<\kappa^{2}$ (i.e., supersonic wave numbers) contribute to sound power radiation; other components are associated with reactive near-field radiation loadings. ${ }^{11}$

The sound pressure in the radiated acoustic far field can also be evaluated from the wave-number transform of plate acceleration using the method of stationary phase ${ }^{9}$ as

$$
\begin{aligned}
p(r, \theta, \phi) & =\frac{\rho e^{j \kappa r}}{2 \pi r} \tilde{w}\left(\kappa_{x}, \kappa_{y}\right)=j \frac{\omega \rho e^{j \kappa r}}{2 \pi r} \widetilde{V}\left(\kappa_{x}, \kappa_{y}\right) \\
& =-\frac{\rho \omega^{2} e^{j \kappa r}}{2 \pi r} \sum_{m=1}^{\infty} \sum_{n=1}^{\infty} W_{m n} V_{m n}\left(\bar{\kappa}_{x}, \bar{\kappa}_{y}\right),
\end{aligned}
$$

where the points of stationary phase are

$$
\begin{aligned}
& \bar{\kappa}_{x}=\sin \theta \cos \phi, \\
& \bar{\kappa}_{y}=\sin \theta \cos \phi .
\end{aligned}
$$

One can show that Eq. (34) is equivalent to Eq. (11). ${ }^{9}$

\section{RESULTS}

For brevity, this paper only considers response of the plate of thickness $2 \mathrm{~mm}$ near the $(3,1)$ response frequency. Table I gives the physical properties of the rectangular simply supported plate considered here, while Table II provides the associated natural frequencies of the lower order modes. For simplicity, a single harmonic point force of amplitude $F=1 N$ located at $x_{f}=0.3163 \mathrm{~m}, y_{f}=0.15 \mathrm{~m}$ was used as the noise disturbance. Likewise, a single control piezoelectric actuator of thickness $t_{a}=0.1905 \mathrm{~mm}$ and dielectric constant of $d_{31},=166 \times 10^{-12} \mathrm{~m} / \mathrm{V}$ was employed, centrally located at $x_{1}=0.158 \mathrm{~m}, x_{2}=0.222 \mathrm{~m}, y_{1}=0.131 \mathrm{~m}$, $y_{2}=0.169 \mathrm{~m}$. A single error sense was assumed located at either $R, \theta, \phi=\left(1.8 \mathrm{~m}, 0^{\circ}, 0^{\circ}\right)$ denoted mike \# 1 or $(1.8 \mathrm{~m}$, $50^{\circ}, 0^{\circ}$ ) denoted mike \#2.

Both pressure and the normal time-averaged intensity (i.e., the $z$ component) to the $x-y$ plane were calculated at a distance of $20 \mathrm{~mm}$ above the plate and expressed in $\mathrm{dB}$ re: 20 $\mu \mathrm{Pa}$ for pressure and $\mathrm{dB}$ re: $10^{-12} \mathrm{~W} / \mathrm{m}^{2}$ for intensity. In addition, the vector intensity components in $x-z$ plane (i.e., vector sum of the $z$ and $x$ component) located at the central line of the plate in the $y$ direction (i.e., $y=L_{y} / 2$ ) as well as the far-field radiation directivity in the same plane at a distance of $R=1.8 \mathrm{~m}$ were calculated. A $k$-plane analysis of the plate response was performed and was plotted in components of $\kappa_{x}$ and $\kappa_{y}$ i.e., wave-number components along the $x$ and $y$ axis, respectively.

The near-field pressure was obtained directly by integrating Eq. (11) using the Simpson's one-third rule approach. These pressure values were then used in the finite difference calculations of the intensity equations with a spacing of $1 \mathrm{~mm}$. The modal sums in the above equations were

TABLE I. Plate specifications.

\begin{tabular}{ccc}
\hline \hline$E=207 \times 10^{9}\left(\mathrm{~N} / \mathrm{m}^{2}\right)$ & $\mu=0.292$ & $L_{x}=0.38(\mathrm{~m})$ \\
$\rho_{p}=7870\left(\mathrm{~kg} / \mathrm{m}^{3}\right)$ & $h=2(\mathrm{~mm})$ & $L_{y}=0.30(\mathrm{~m})$ \\
\hline \hline
\end{tabular}

TABLE II. Natural frequencies of simply supported plate $(\mathrm{Hz})$.

\begin{tabular}{cc}
\hline \hline Mode $(m, n)$ & $f_{m n}$ \\
\hline$(1,1)$ & 87.71 \\
$(2,1)$ & 188.74 \\
$(1,2)$ & 249.81 \\
$(2,2)$ & 350.85 \\
$(3,1)$ & 357.13 \\
$(3,2)$ & 519.23 \\
$(1,3)$ & 519.98 \\
$(4,1)$ & 592.88 \\
\hline
\end{tabular}

truncated at $m=n=5$, i.e., 25 modes were included in the analysis. This number of modes was found to provide sufficient accuracy for the study of the behavior of the system before and after control.

\section{A. Pressure and intensity distributions}

Figure 3 presents the far-field radiation directivity patterns for a frequency of $357 \mathrm{~Hz}$, which can be seen from Table II to be close to the $(3,1)$ resonance point. The solid line denotes the noise field and can be seen to be fairly constant with radiation angle. This behavior is due to the relatively long wavelength of the acoustic radiation relative to plate size, leading to the higher order plate giving a radiation field which is volumetric or monopole like.

The controlled field is shown as a dashed line when the error sensor is located at $\theta=0^{\circ}$. The results show that the noise field is strongly attenuated globally, and the residual field exhibits a dipole like radiation pattern due to the position of the error microphone. When the error microphone is moved to $\theta=50^{\circ}$, similar values of attenuation are achieved. However, the null in the residual radiation field has now moved to location of the error microphone.

Although the different location of error microphone results in different residual radiation pattern, the optimal voltages peak-to-peak applied to the piezoelectric actuator are almost identical for the error microphone at $\theta=0^{\circ}$ and $50^{\circ}$ (24.96 and $24.93 \mathrm{~V}$, respectively), and the total acoustic
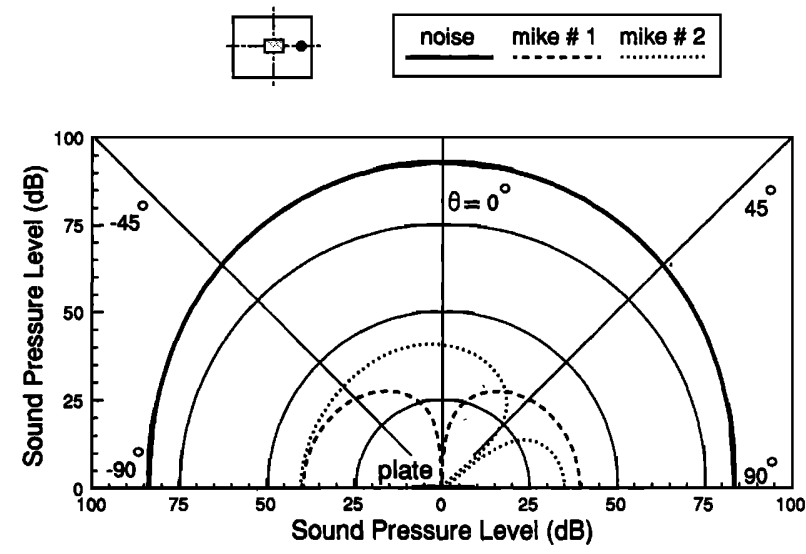

FIG. 3. Radiation directivity, $f=357 \mathrm{~Hz}$. 

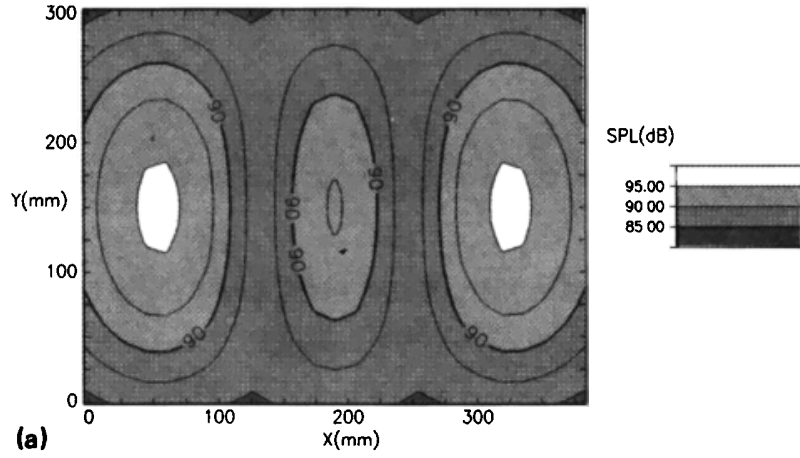

(a)
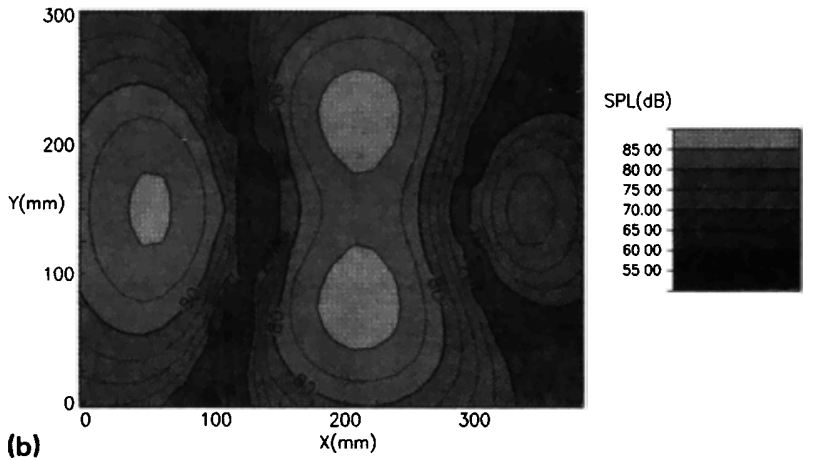

(b)

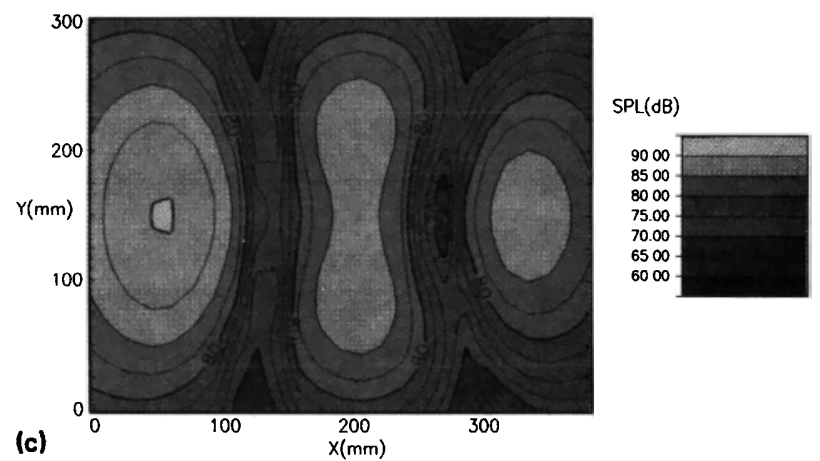

FIG. 4. (a) Pressure distribution ( $x y$ plane) for the noise source only, $f=357 \mathrm{~Hz}$. (b) Pressure distribution ( $x y$ plane) with control, $f=357 \mathrm{~Hz}$, error mike at $(R, \theta, \phi)=\left(1.8 \mathrm{~m}, 0^{\circ}, 0^{\circ}\right)$. (c) Pressure distribution ( $x y$ plane) with control, $f=357 \mathrm{~Hz}$, error mike at $(R, \theta, \phi)=\left(1.8 \mathrm{~m}, 50^{\circ}, 0^{\circ}\right)$.

power reduction achieved in the two cases are $57.18 \mathrm{~dB}$ for $\theta=0^{\circ}$ and $51.68 \mathrm{~dB}$ for $\theta=50^{\circ}$ respectively. As seen in Fig. 3 , the sound pressure level of the uncontrolled field at $\theta=0^{\circ}$ is higher than that at $\theta=50^{\circ}$. Although for the single-input, single-output system the error microphone signal is driven to zero, the use of the error microphone at $\theta=0^{\circ}$ results in more overall pressure reduction than that at $\theta=50^{\circ}$. Therefore, the error microphone at $\theta=0^{\circ}$ perform slightly better than that at $\theta=50^{\circ}$.

The corresponding near-field pressure distributions in the $x-y$ plane for the noise and controlled (error microphone at $\theta=0^{\circ}$ ) fields are shown in Fig. 4(a) and (b), respectively. The noise field can be seen to exhibit the cell like behavior associated with the $(3,1)$ mode shape of the plate. When control is applied, two changes in the near-field pressure distribution of Fig. 4(b) are observed. Firstly, the overall pressure amplitudes are reduced by around $10 \mathrm{~dB}$. Secondly,
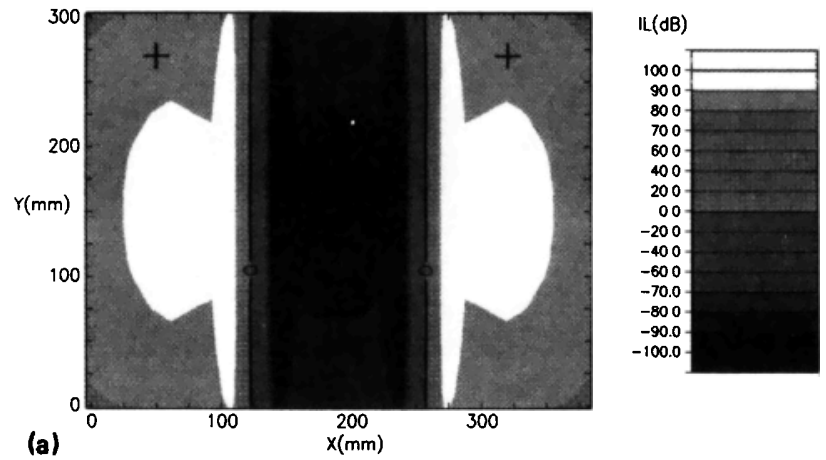

(a)

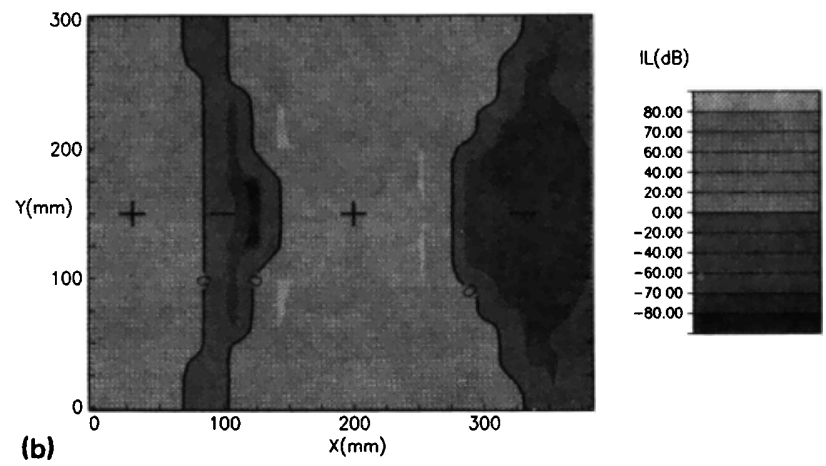

(c)
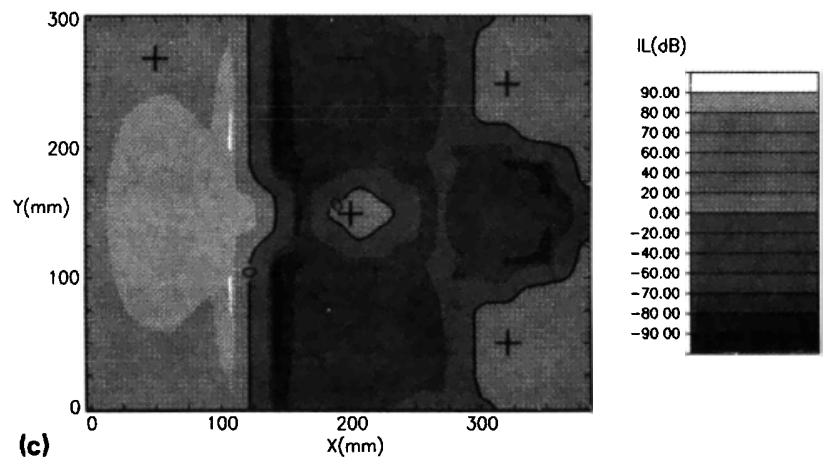

FIG. 5. (a) Time-averaged intensity distribution ( $x y$ plane) for the noise source only, $f=357 \mathrm{~Hz}$. (b) Time-averaged intensity distribution ( $x y$ plane) with control, $f=357 \mathrm{~Hz}$, error mike at $(R, \theta, \phi)=\left(1.8 \mathrm{~m}, 0^{\circ}, 0^{\circ}\right)$. (c). Time-averaged intensity distribution ( $x y$ plane) with control, $f=357$ $\mathrm{Hz}$, error mike at $(R, \theta, \phi)=\left(1.8 \mathrm{~m}, 50^{\circ}, 0^{\circ}\right)$.

the complexity of the pressure field is markedly increased, and there appears to be a semblance of a pressure node located around $x=100 \mathrm{~mm}$. It is this kind of behavior that is interesting for the design of near-field sensors. The overall fall in near-field pressure indicates that if a distributed pressure sensor is located above and completely covers the plate, then minimizing the near-field sensor output might have the same control influence as a far-field point sensor, at least for plate modes on resonances. In effect the use of a large distributed pressure sensor located near the plate may lead to an "unloading" of the plate radiation field by causing a large area of low pressure on or near the surface of the plate. The overall result would be a drop in radiated power due to the low radiation impedance seen by the plate.

Figure 5(a) and (b) give the corresponding normal intensity distributions to Fig. 4(a) and (b). Regions of outgoing and ingoing intensity to the plate are marked $(+)$ and 
( - ), respectively. Note also in the scale that negative intensity indicates intensity flowing into the plate not negative intensity level. The noise field of Fig. 5(a) strongly indicates the $(3,1)$ plate behavior. As can be seen, the two outer cells give positive or outgoing intensity, while the inner cell has negative or in-going intensity. However, as discussed in previous works, such as by Maidanik, ${ }^{12}$ the net effect for long acoustic wavelength relative to plate size is edge radiation, which appears like a monopole type source in the far field. As the acoustic wavelengths are relatively long, radiation of each half cell interacts with its neighbor leading to cancellation for the inner portions of the plate. When control is applied the intensity distribution is markedly changed. Figure 5(b) shows that the apparent plate source is far more complex and higher order. An integration of the intensity vector over the plate surface shows that the net power has been strongly attenuated. Since the single actuator can suppress strongly responding modes [in this case the $(3,1)$ mode], the residual plate modes are usually higher order. It is observed that both the controlled pressure and intensity fields of Figs. 4(b) and 5(b) have a shape very similar to the $(4,1)$ mode. This higher mode pattern has lower radiation efficiency, and this, in addition to the reduction in amplitude, leads to a drop in radiated pressure. The first effect has been called "modal suppression" and the second "modal restructuring." 13

Figures 4(c) and 5(c) show the controlled pressure field and normal intensity distribution when the error microphone is located at $\theta=50^{\circ}$. The near-field pressure field appears to be fairly similar to the distribution when the error microphone was located at $\theta=0^{\circ}$ given in Fig. 4(b). However, the intensity distribution is markedly different. In this case the central cell in which intensity flows into the plate, has greatly expanded, while the edge radiation components appear to have been reduced. In particular, the net power flow from the edge of the plate on the same side as the error microphone has been markedly attenuated.

Figures 6(a) and 7(a) present the near-field pressure distribution and intensity vector distribution in the central $x-z$ plane for the noise source. For the intensity plots a calibrated vector length is provided for scaling purposes in the bottom right hand corner. Note that the intensity vector plots are in $\log$ scale. The pressure distributions clearly illustrate the volumetric radiation nature of the $(3,1)$ mode at this low value of $\kappa L_{x}=2.48$. Near the plate, the pressure distribution exhibits the three cell nature associated with the $(3,1)$ mode; however, as the observation point moves to the far field, the radiation field becomes progressively more uniform and monopole like in behavior. Similarly, the intensity distributions show that the inner cell of the plate acts as an acoustic sink, and the radiated energy from the plate comes from near the edges.

When control is applied, the pressure and intensity distributions for the error microphone at $\theta=0^{\circ}$ demonstrate a marked change as shown in Figs. 6(b) and 7(b). Two characteristics are again evident. Firstly, the overall pressure levels have fallen (due to response of the plate decreasing). Close to the plate, the pressure field exhibits behavior like the $(3,1)$ mode; however, it is apparent that strong nodal
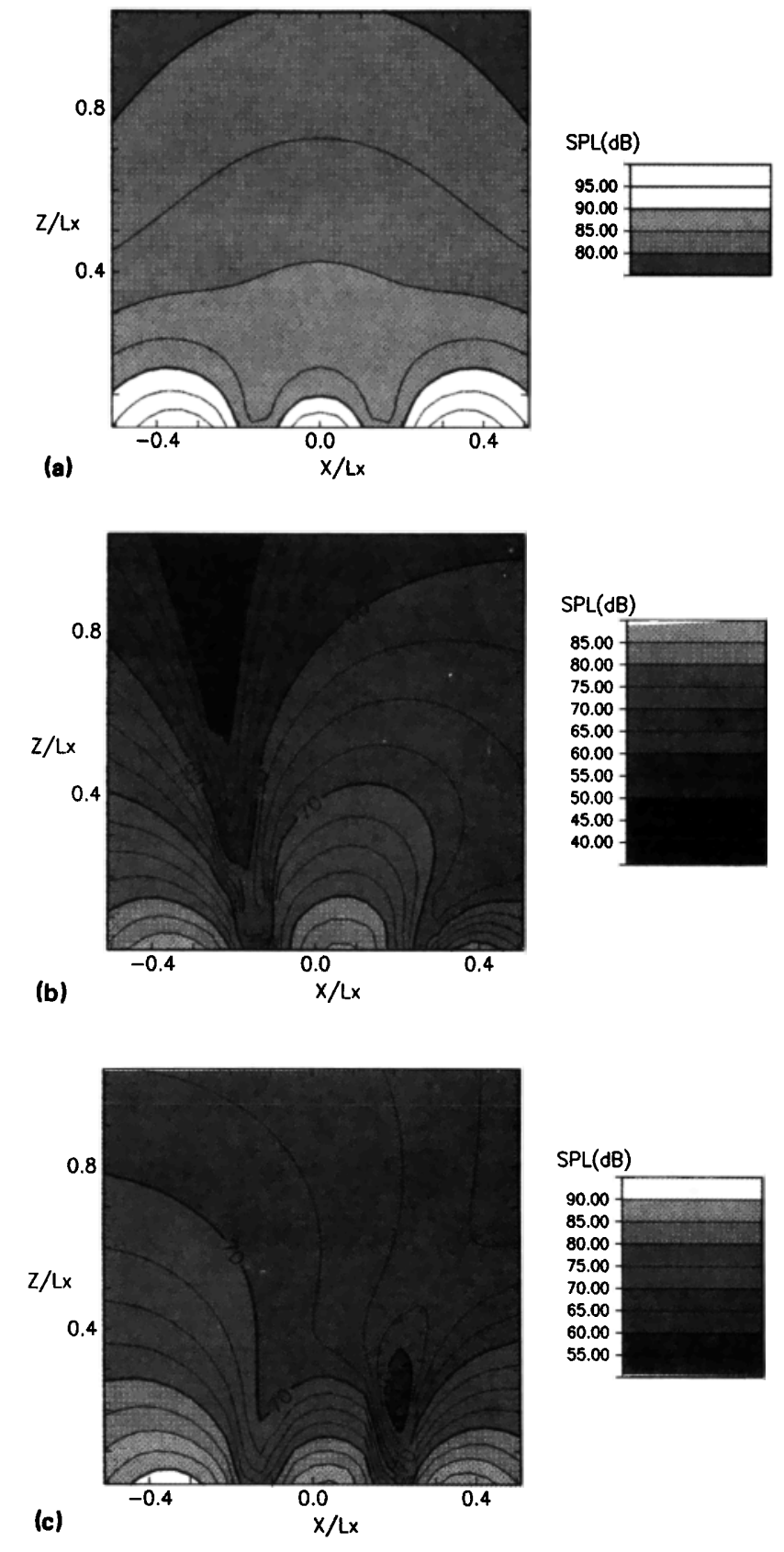

FIG. 6. (a) Pressure distribution ( $x z$ plane) for the noise source only, $f=357 \mathrm{~Hz}$. (b) Pressure distribution ( $x z$ plane) with control, $f=357 \mathrm{~Hz}$, error mike at $(R, \theta, \phi)=\left(1.8 \mathrm{~m}, 0^{\circ}, 0^{\circ}\right)$. (c) Pressure distribution ( $x z$ plane) with control $f=357 \mathrm{~Hz}$, error mike at $(R, \theta, \phi)=\left(1.8 \mathrm{~m}, 50^{\circ}, 0^{\circ}\right)$.

type region of low pressure has been generated starting near $x / L_{x}=0.4$. Likewise, the intensity vector distribution for this case given in Fig. 7(b) show that the net radiation from the edges has been effectively canceled. It is interesting to note from Fig. 7(b) that the noise source appears to be acting as an energy sink in this case.

Moving the error microphone to $\theta=50^{\circ}$ gives additional interesting results, as can be seen in the pressure plots of Fig. 6(c) and intensity vector plots of Fig. 7(c). Again, the controlled pressure field exhibits behavior very similar to the $(3,1)$ mode near the plate without large changes in level; however, at increasing distances from the plate, the pressure field becomes increasingly more complex exhibiting a nodaltype low-pressure region towards the location of the error 

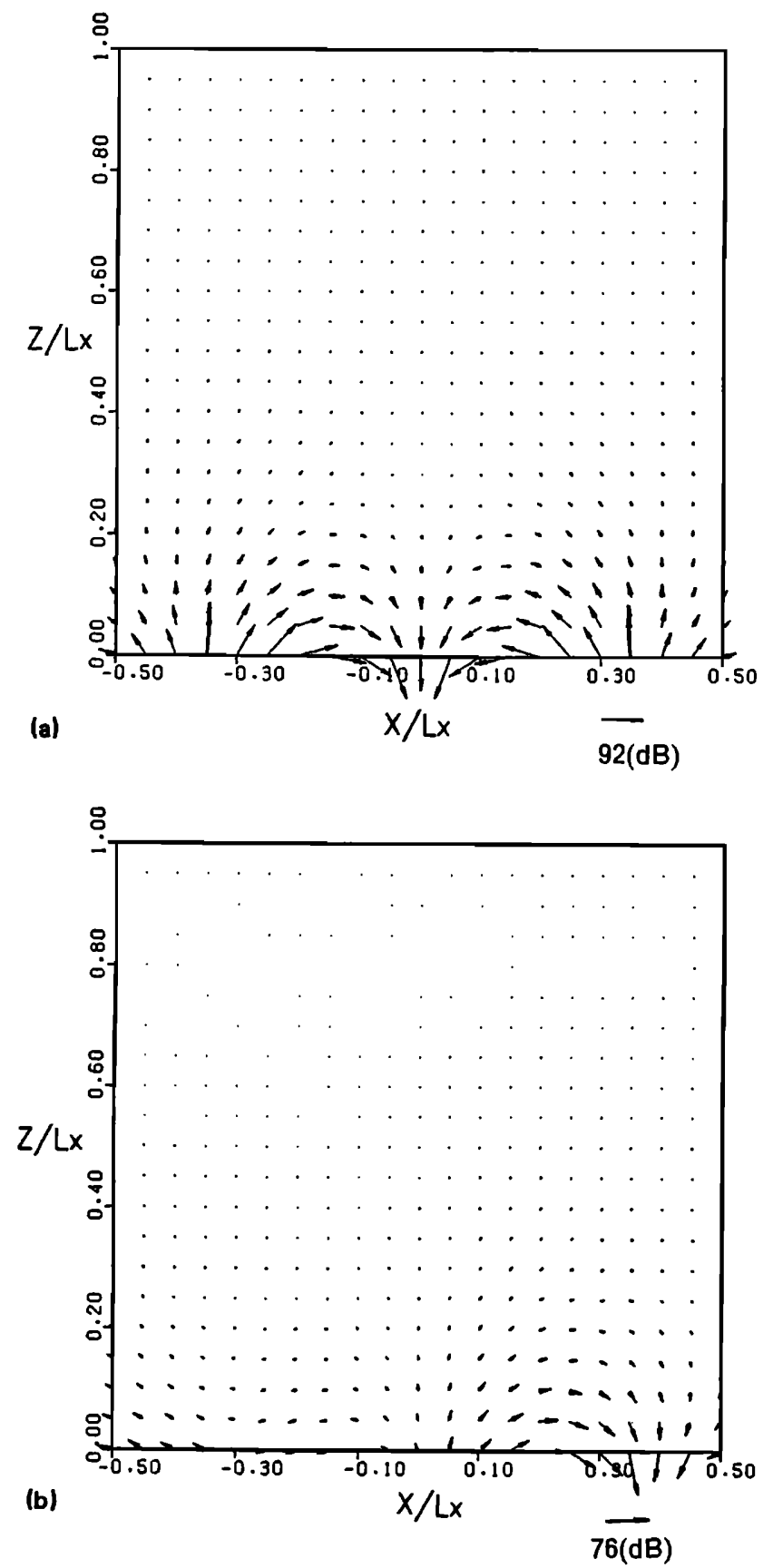

microphone. The intensity plots of Fig. 7(c) again demonstrate that the edge radiation is effectively reduced, this time on the side of the plate closest to the error microphone. In this case, it appears that the energy leaving one side of the plate flows across the plate surface and is absorbed at the other side.

Thus two important effects have been illustrated apart from the fact that control leads to a general lowering of radiation acoustic pressure. The results demonstrate that application of control leads to a change in the volumetric, monopole nature of the $(3,1)$ mode in the far field and also a reduction in the edge radiation component.

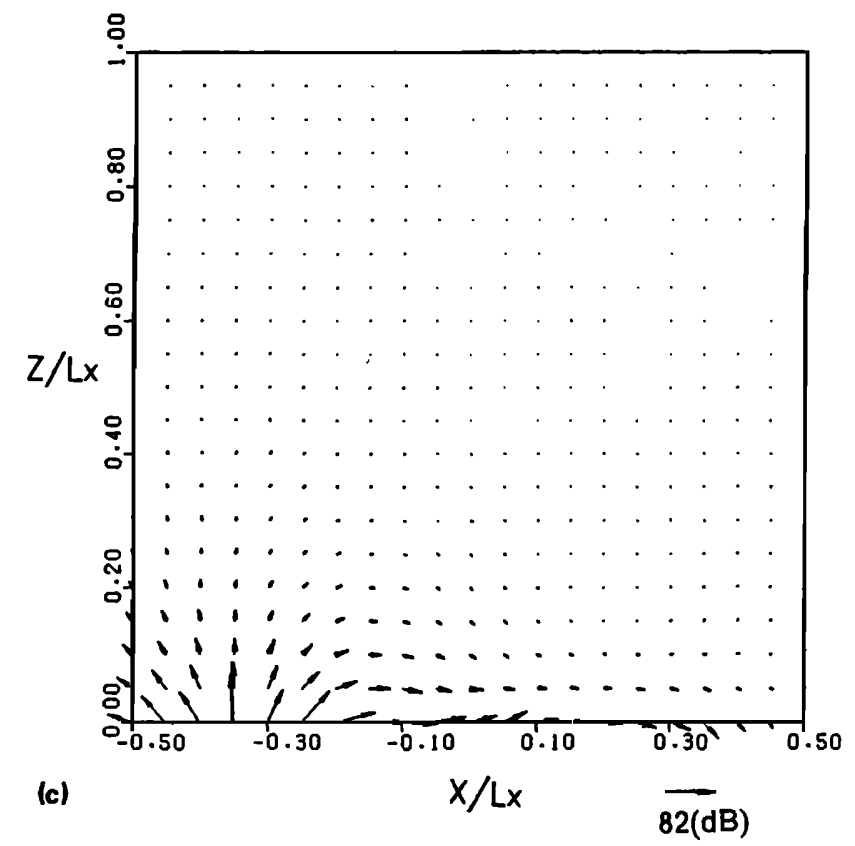

FIG. 7. (a) Intensity vector ( $x z$ plane) for the noise source only, $f=357$ Hz. (b) Intensity vector ( $x z$ plane) with control, $f=357 \mathrm{~Hz}$, error mike at $(R, \theta, \phi)=\left(1.8 \mathrm{~m}, 0^{\circ}, 0^{\circ}\right)$. (c) Intensity vector ( $x z$ plane) with control, $f=357 \mathrm{~Hz}$, error mike at $(R, \theta, \phi)=\left(1.8 \mathrm{~m}, 50^{\circ}, 0^{\circ}\right)$.

\section{B. Wave-number distributions}

In connection with the last observation, it is interesting to examine the wave-number spectrum of the plate uncontrolled and controlled response. Figure 8(a) and (b) gives the modulus squared (the autospectrum) of the plate wavenumber components along $\kappa_{x}$ and $\kappa_{y}$ axis, respectively. It is noted that only the positive components of $\kappa_{x}$ and $\kappa_{y}$ are shown in Fig. 8(a) and (b) for brevity; however, the complete plate wave-number spectrum should also include negative components. As the modes on the plate are separable, we can completely represent the plate wave-number distribution by these plots, and it is noted that $\kappa_{p}^{2}=\kappa_{x}^{2}+\kappa_{y}^{2}$. 


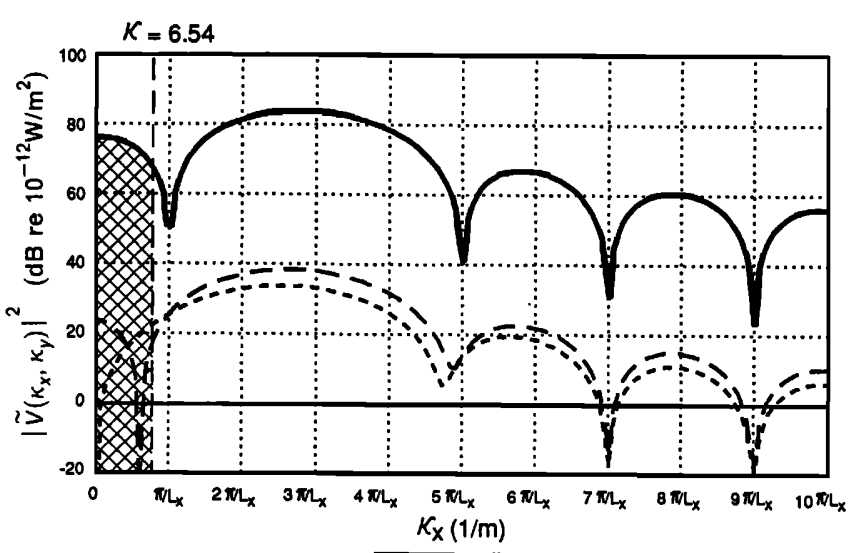

(a)

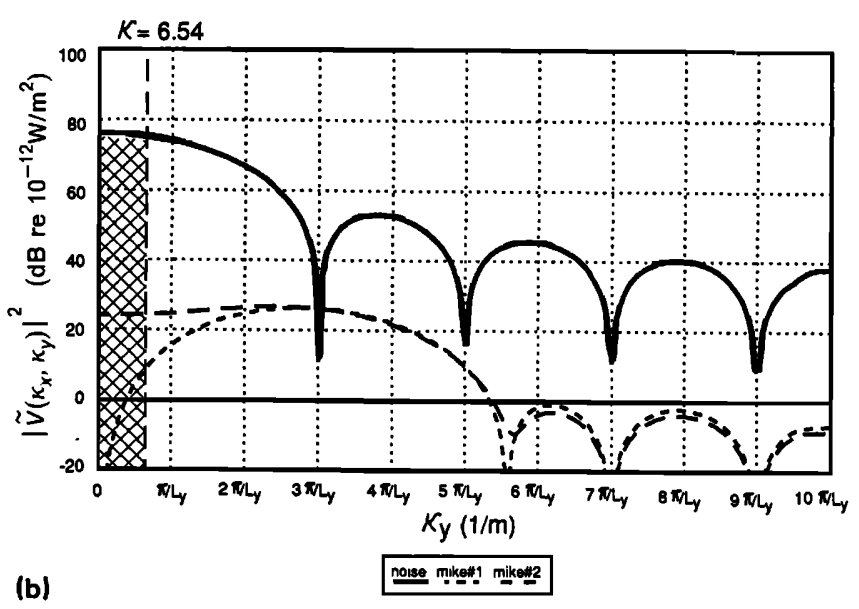

FIG. 8. (a) Wave-number spectra of plate modal velocity along $\kappa_{x}$ axis. (b) Wave-number spectra of plate modal velocity along $\kappa_{y}$ axis.

In Fig. 8(a) and (b), the solid line denotes the spectrum of the noise field, while the short-dash line represents the controlled field for the error microphone at $\theta=0^{\circ}, \phi=0^{\circ}$ and the long-dash line for the case of the error microphone at $\theta=50^{\circ}, \phi=0^{\circ}$. The spectrum of the noise field can be seen from Fig. 8(a) to peak near values of $\kappa_{x}=3 \pi / L_{x}$ and $\kappa_{y}$ $=0$ indicating that the plate response is dominated by the $(3,1)$ mode whose eigenvalues are $\kappa_{x}=3 \pi / L_{x}, \kappa_{y}=\pi / L_{y}$, respectively. Figure 8(a) also indicates that there is substantial spectral component at $\kappa_{x}=0$ indicating that there is significant $(1,1)$ mode content in the plate response. This observation explains why the pressure and intensity fields of Figs. 4(a) and 5(a) are slightly distorted from the pure $(3,1)$ distribution.

The spectral content of the controlled response can be seen to be strongly reduced at all wave numbers for both microphone positions. This indicates that the plate response has globally fallen explaining the overall drop in near-field pressure observed previously. Also shown on the plots of Fig. 8(a) and (b) is the position of the wave-number component $\kappa_{x},=\kappa_{y}=\kappa$; for this frequency $\kappa=\omega / c=6.54 \mathrm{~m}^{-1}$. As discussed by Maidanik, ${ }^{12}$ wave-number components above this line are subsonic and do not contribute to far-field pressure. However, the supersonic components below $\kappa$ do contribute to the far-field pressure. Thus it is interesting to note a number of further observations from Fig. 8(a). Firstly, the wave-number spectrum can be seen to be zero at $\kappa_{x}$ $=0$ and $\kappa_{x}=5 \mathrm{~m}^{-1}$ for the controlled cases with the error microphone at $\theta=0^{\circ}$ and $50^{\circ}$, respectively. This result supports the stationary phase result of Eqs. (33), (34), and (35), which states that radiation at a particular angle corresponds to discrete wave-number components at $\kappa_{x}=\kappa$ $\times \sin \theta \cos \phi$ and $\kappa_{y}=\kappa \sin \theta \sin \theta$. Thus, when the pressure is minimized in the far field at a particular angle, the corresponding wave-number component is suppressed in the structure. This result suggests that a controller could be formulated in the wave-number domain to remove particular wave-number components from the structure. The radiated pressure would then be minimized at the corresponding angle without the use of a microphone in the farfield. Control formulations of this nature have recently been completed at VPI\&SU. ${ }^{14}$

The second interesting point of Fig. 8(a) is that the supersonic components have also been reduced to a greater degree than the subsonic components. As discussed by Fahy, ${ }^{11}$ the supersonic components arise because of the finiteness of the plate embedded in the baffle (the reader is reminded that the wave-number transform considers the complete plate-baffle system). As discussed previously, these components are thus related to the edge discontinuity of the plate. The phenomena is termed "edge radiation". ${ }^{12}$ Reduction in the supersonic component thus implies a reduction or change in the edge radiation mechanism, and this is precisely the behavior that was observed in the intensity vector plots of Fig. 7(b) and (c) discussed previously.

Finally, the controlled wave-number distributions of Fig. 8(a) and (b) show that the oscillations in the spectral distributions at low wave numbers has been smoothed. This indicates that the residual response of the plate-baffle system is dominated by high wave-number components or short wavelength high modal number motion, as observed in the residual or controlled pressure and normal intensity plots.

Thus wave-number analysis appears to be a powerful alternative tool to investigate the system behavior. For example, what was previously termed "modal suppression," when the plate response falls globally, ${ }^{13}$ corresponds to a fall in wave-number components across the spectrum. What was previously termed "modal restructuring," which corresponds to a change or increase in plate vibration amplitude and complexity while radiated pressure falls, ${ }^{13}$ corresponds to a reduction in supersonic components while the subsonic components may even increase. Both of these types of behavior have been observed in the wave-number domain in companion experiments. ${ }^{15}$

\section{CONCLUSIONS}

This paper presents an analytical study of the near-field pressure and intensity distributions of actively controlled plate-radiated sound. The results presented, although limited to the $(3,1)$ mode resonance case, reveal a number of 
interesting characteristics. In general, when control was applied, a number of observations were made. First, for this case of resonance, applying control leads to an overall fall in the magnitudes of near-field pressure and intensity vectors. Second, the controlled field appeared to exhibit radiation behavior similar to a higher-order antisymmetric mode. The monopole like nature of the far-field radiation pattern of the $(3,1)$ mode was modified to a pattern with a nodal region near the location of the error microphone. Third, the application of control appeared to reduce the acoustic intensity leaving the edges of the plate (edge radiation) resulting in an overall drop in radiated acoustic power. In connection with this, while the location of the error mike had little significant effect on the overall power reduction and optimal control voltages, it had a significant effect on the near-field pressures and intensity distributions.

The results provide further insight into the mechanisms associated with the active structural acoustic control (ASAC) approach studied here. In particular, the results indicate possible strategies for the design of near-field sensors to minimize the far-field radiated power. For "on resonance" case, one strategy may be to use a distributed sensor which provides an error signal proportional to the average of the acoustic pressure over the plate surface. Another strategy could be to use a strip distributed error sensor that could be located at the very low pressure regions of the controlled distribution. This would have the effect of forcing an asymmetry into the radiation field which has been shown in this paper to be associated with a lower total radiated acoustic power. Situations such as these are presently under theoretical and experimental investigation at VPI\&SU. However, this work only considers one particular operating case, and much further work, particularly for off-resonance conditions, is needed to design and implement reliable near-field sensors based upon the above observations. Finally, wavenumber analysis of the plate vibration has been shown to be a powerful alternative tool for studying the mechanisms of control.

\section{ACKNOWLEDGMENTS}

The authors gratefully acknowledge the support of this research by DARPA and by the Office of Naval Research under grant ONR-N00014-88-K-0721.

'B. T. Wang, C. R. Fuller, and E. K. Dimitriadis, "Active Control of Noise Transmission Through Rectangular Plates Using Multiple Piezoelectric or Point Force Actuators," J. Acoust. Soc. Am. 90, 2820-2830 (1991).

${ }^{2}$ B.-T. Wang, E. K. Dimitriadis, and C. R. Fuller, "Active Control of Structurally Radiated Noise Using Multiple Piezoelectric Actuators," AIAA J. 29, 1771-1776 (1991).

${ }^{3}$ O. K. O. Pettersen, "A Procedure for Determining the Sound Intensity Distribution Close to a Vibrating Surface," J. Sound Vib. 66(4), 626-629 (1979).

${ }^{4}$ G. Krishnappa and J. M. McDougall, "Sound Intensity Distribution and Energy Flow in the Nearfield of a Clamped Circular Plate," J. Vib. Acoust. Stress Reliability Design 111, 465-471 (1989).

${ }^{5}$ U. R. Kristiansen, "A Numerical Study of the Acoustic Intensity Distribution Close to a Vibrating Membrane," J. Acoust. Soc. Am. 76, 305-309 (1981).

${ }^{6} \mathbf{J}$. K. Thompson and D. R. Tree, "Finite Difference Approximation Errors in Acoustic Intensity Measurements," J. Sound Vib. 75(2), 229-238 (1981).

${ }^{7}$ E. K. Dimitriadis, C. R. Fuller, and C. A. Rogers, "Piezoelectric Actuators for Distributed Vibration Excitation of Thin Plates," J. Vib. Acoust. 113, 100-107 (1991).

${ }^{8}$ L. A. Roussos, "Noise Transmission Loss of a Rectangular Plate in an Infinite Baffle," NASA Tech. Paper 2398, March (1985).

${ }^{9}$ M. C. Junger and D. Feit, Sound, Structures and their Interaction (MIT Press, Cambridge, MA, 1986), 2nd ed., pp. 112-116.

${ }^{10} \mathrm{H}$. C. Lester and C. R. Fuller, "Active Control of Propeller Induced Noise Fields Inside a Flexible Cylinder," AIAA J. 28(8), 1374-1380 (1990).

"F. Fahy, Sound and Structural Vibration: Radiation, Transmission and Response (Academic, Orlando, FL, 1985), pp. 72-89.

${ }^{12} \mathrm{G}$. Maidanik, "Vibrational and Radiative Classifications of Modes of a Baffled Finite Panel," J. Sound Vib. 34(4), 447-455 (1974).

${ }^{13}$ C. R. Fuller, C. H. Hansen, and S. D. Snyder, "Active Control of Sound Radiation From a Vibrating Rectangular Panel by Sound Sources and Vibration Inputs: An Experimental Comparison," J. Sound Vib. 145(2), 195-215 (1991).

${ }^{14}$ C. R. Fuller and R. A. Burdisso, "A Wave Number Domain Approach to the Active Control of Sound and Vibration," J. Sound Vib. 148(2), 355360 (1991).

${ }^{15}$ R. L. Clark and C. R. Fuller, "Modal Sensing of Efficient Acoustic Radiators with PVDF Distributed Sensors in Active Structural Acoustic Control Approaches," J. Acoust. Soc. Am. 91, 3321-3329 (1992). 